\title{
Influência do tráfego de veículos no teor de alumínio e metais pesados em infusão de macela (Achyrocline satureioides Lam DC.)
}

\author{
Natiele da Silva GALVAN ${ }^{1 *}$, Mari Lucia CAMPOS ${ }^{1}$, David José MIQUELLUTI ${ }^{1}$, \\ Andrei de Souza da SILVA ${ }^{1}$ \\ ${ }^{1}$ Centro de Ciências Agroveterinárias, Universidade do Estado de Santa Catarina, Lages, SC, Brasil. \\ *E-mail: natiele.galvan@gmail.com
}

Recebido em dezembro/2018; Aceito em setembro/2019.

\begin{abstract}
RESUMO: Registros etnofarmacológicos indicam diversas propriedades terapêuticas atribuídas à decocção das inflorescências de macela, como, por exemplo, eficaz no combate a alta pressão arterial e alto colesterol, cólicas de origem nervosa, para limpeza de feridas, diarréia, como anti-inflamatória e distúrbios gastrointestinais. A macela na sexta-feira Santa é coletada próximo a rodovias e pode aumentar o risco de contaminação humana com metais pesados. Os metais $\mathrm{Cd}, \mathrm{Cu}, \mathrm{Pb}$ e $\mathrm{Zn}$ são liberados durante as diferentes operações de transporte rodoviário, tais como a combustão, desgaste dos pneus, vazamento de óleos, desgaste de pastilhas e discos de frenagem, radiadores, etc. O presente estudo tinha como objetivo quantificar os teores de $\mathrm{Al}$ e de metais pesados como, $\mathrm{Cu}$, $\mathrm{Mn}, \mathrm{Ni}, \mathrm{Pb}$ e Zn na infusão de macela coleta em locais com influência de tráfego rodoviário. As flores de macela foram coletadas as margens das rodovias BR- 282; SC- 390 e SC- 114 e respectivas áreas testemunhas (sem tráfego), separadas dos ramos e secas em estufa de circulação a $65^{\circ} \mathrm{C}$. O processo de infusão foi realizado segundo protocolo de Anderson (1999), sendo que, a quantificação de Al, Cu, Mn, Ni e Zn em ICP-OES e Pb em HR-CS AAS. Em média as infusões das amostras de macela coletadas as margens da BR 282 apresentaram os maiores teores de $\mathrm{Al}, \mathrm{Cu}, \mathrm{Ni}, \mathrm{Mn}$ e $\mathrm{Zn}$. Não houve diferença estatística entre teores de $\mathrm{Pb}$ em macelas coletadas nas diferentes rodovias e as testemunhas. O consumo de chá de macela coletada próximo a rodovias pode resultar em risco a saúde humana.
\end{abstract}

Palavras-chave: chá; elementos tóxicos; tráfego rodoviário.

\section{Influence of vehicle traffic in al and heavy metals in macela infusion (Achyrocline satureioides Lam DC.)}

\begin{abstract}
Ethnopharmacological records indicate several therapeutic properties attributed to the decoction of macela inflorescences, such as, for example, effective in combating high blood pressure and high cholesterol, cramps of nervous origin, for wound cleaning, diarrhea, as well as anti-inflammatory and gastrointestinal disorders. The macela on Good Friday is collected near highways and may increase the risk of human contamination with heavy metals. The $\mathrm{Cd}, \mathrm{Cu}, \mathrm{Pb}$ and $\mathrm{Zn}$ metals are released during different road transport operations, such as combustion, tire wear, oil leakage, wear of brake pads and discs, radiators, etc. The objective of this study was to quantify the levels of $\mathrm{Al}$ and heavy metals such as $\mathrm{Cu}, \mathrm{Mn}, \mathrm{Ni}, \mathrm{Pb}$ and $\mathrm{Zn}$ in the infusion of macela collected at sites influenced by road traffic. The macela flowers were collected along the margins of the BR-282 highways; SC-390 and SC-114 and their control areas (without traffic), separated from the branches and dried in a circulation oven at $65^{\circ} \mathrm{C}$. The infusion process was performed according to Anderson's protocol (1999), and quantification of $\mathrm{Al}, \mathrm{Cu}, \mathrm{Mn}, \mathrm{Ni}$ and $\mathrm{Zn}$ in ICP-OES and $\mathrm{Pb}$ in HR-CS AAS. On average the infusions of the macela samples collected at the borders of BR 282 presented the highest levels of $\mathrm{Al}, \mathrm{Cu}, \mathrm{Ni}, \mathrm{Mn}$ and $\mathrm{Zn}$. There was no statistical difference between $\mathrm{Pb}$ contents in the macela collected on the different highways and the witnesses. The consumption of macela tea collected near highways can result in a risk to human health.
\end{abstract}

Keywords: tea; toxic elements; road traffic.

\section{INTRODUÇÃO}

Infusões das inflorescências da macela (Achyrocline satureioides) são utilizadas na medicina popular brasileira como um agente digestivo, antiespasmódico, anti-inflamatório e hipoglicêmico, para tratar desordens gastrintestinais e reduzir os níveis de colesterol sanguíneo (RITTER et al., 2002; SIMÕES et al., 1988).

A composição química de macela foi extensivamente estudada por diversos pesquisadores, sendo relatada a presença de monoterpenóides do óleo essencial; ácidos cafeico, clorogênico e isoclorogênico e seus ésteres; outros terpenóides, compostos acetilênicos, derivados da kawapirona, polissacarídeos e minerais (AKISUE, 1971; BOHLMANN et al., 1973; BROUSSALIS et al., 1988; LAMATY et al; 1991; PETROVICK et al., 1997).

A fração etanólica do extrato obtido das inflorescências da macela apresenta flavonoides quercetina, 3-Ometilquercetina e luteolina (FERRARO et al., 1981; SIMÕES et al., 1988) que se relacionam com os principais efeitos farmacológicos atribuídas a esta planta.

Registros etnofarmacológicos indicam diversas propriedades terapêuticas atribuídas à decocção das 
inflorescências de macela, como, por exemplo, eficaz no combate a alta pressão arterial e alto colesterol (KOCH, 2000), epilepsia, cólicas de origem nervosa, infecção intestinal e para limpeza de feridas (PIRKER et al., 2012), diarreia (BRANDELLI et al., 2009), como anti-inflamatória e distúrbios gastrointestinais (SIMÕES et al., 1988).

Existe uma tradição religiosa em relação à época da colheita da macela, na região sul do Brasil. Ela é colhida na madrugada da sexta-feira Santa no nascer do sol. Os fiéis acreditam que o orvalho que cai sobre os arbustos na Sextafeira que antecede a Páscoa faz com que a planta seja abençoada, podendo causar até curas milagrosas (DIÁRIO CATARINENSE, 2013). Nessa época do ano a coleta da planta é realizada no campo e nas circunvizinhanças de estradas vicinais e rodovias. A coleta de macela próximo a rodovias pode aumentar o risco de contaminação humana com metais pesados.

Rodovias e estradas intensamente trafegadas são importantes fontes de poluentes introduzidos no meio ambiente. O crescente volume de tráfego mundial leva ao aumento das taxas de emissão, causando uma contaminação dos solos a margem (MONKS et al., 2009).

Os poluentes derivados do tráfego originam-se de uma variedade de fontes, incluindo combustão incompleta e completa de combustível, perdas de combustível e vazamento de óleo de sistemas hidráulicos. Corrosão, atividades de degelo e desgaste de componentes de veículos (pneus, freios, embreagem e motor) também são fontes importantes de emissões de tráfego (HJORTENKRANS et al., 2007, MCKENZIE et al., 2009).

A composição e a quantidade de deposição seca e úmida dependem de muitos fatores, como a intensidade do tráfego (WARD, 1999; ARSLAN et al., 2006), direção e velocidade do vento do vento, intensidade e períodos de chuva, períodos de seca anteriores, projeto de estradas e da cobertura vegetal. (BARBOSA; HVITVED-JACOBSEN, 1999; PAGOTTO et al., 2001; LEE et al., 2011).

A poluição dos solos por metais pesados de fonte automotiva é um grave problema ambiental em todo o mundo
(AKBAR et al., 2006; DOLAN et al., 2006; BAKER et al., 2007; YOSHINORI et al., 2010). Os metais $\mathrm{Cd}, \mathrm{Cu}, \mathrm{Pb}$ e $\mathrm{Zn}$ são libertados durante as diferentes operações de transporte rodoviário, tais como a combustão, desgaste dos pneus, vazamento de óleos, desgaste de pastilhas e discos de frenagem, radiadores, etc. (AKBAR et al., 2006; DOLAN et al., 2006; BAKER et al., 2007; YOSHINORI et al., 2010; LOUGH et al., 2005; PEREIRA et al., 2010). Após a avaliação de 27 estudos, num total de 64 locais distribuídos por toda a Europa, Werkenthin et al. (2014) observaram altos teores de $\mathrm{Cr}, \mathrm{Cu}, \mathrm{Ni}, \mathrm{Pb}$, e $\mathrm{Zn}$ na camada de $0-20 \mathrm{~cm}$, nos primeiros $5 \mathrm{~m}$ de distância das rodovias. Esses autores também observaram que os teores diminuem com o aumento da distância entre o ponto coletado e a rodovia.

O chá de macela é popularmente utilizado na região sul do Brasil como fitoterápico, porém, não há estudos sobre a influência do tráfego de veículos e os teores de metais pesados. Neste contexto, o presente estudo teve como objetivo quantificar os teores de $\mathrm{Al}$ e de metais pesados como, $\mathrm{Cu}, \mathrm{Mn}$, $\mathrm{Ni}, \mathrm{Pb}$ e $\mathrm{Zn}$ na infusão de macela coleta em locais com influência de tráfego rodoviário.

\section{MATERIAL E MÉTODOS}

\subsection{Coleta}

As amostras de plantas foram coletadas entre o dia 20 de março de 2017 e 31 de março de 2017 nos Municípios de Capão Alto, Lages e Palmeira no estado de Santa Catarina. Em cada um dos municípios foram definidos um local de coletas com influência de tráfego rodoviário $(\mathrm{P} 1)$ e uma testemunha sem tráfego (P2) (Figura 1). Em cada local foram coletadas oito amostras, totalizando quarenta e oito amostras de plantas.

As amostras com influência de tráfego rodoviário foram coletadas nas rodovias SC 390, BR - 282 e SC - 114. As SC 390 e SC - 114 possuem tráfego de veículos leves e pesados menor que a BR 282. A rodovia BR 282 foi construída para suportar um tráfego de cinco mil veículos diários, atualmente, tem um tráfego de mais de 20 mil (SANTA CATARINA 24 HORAS, 2018).

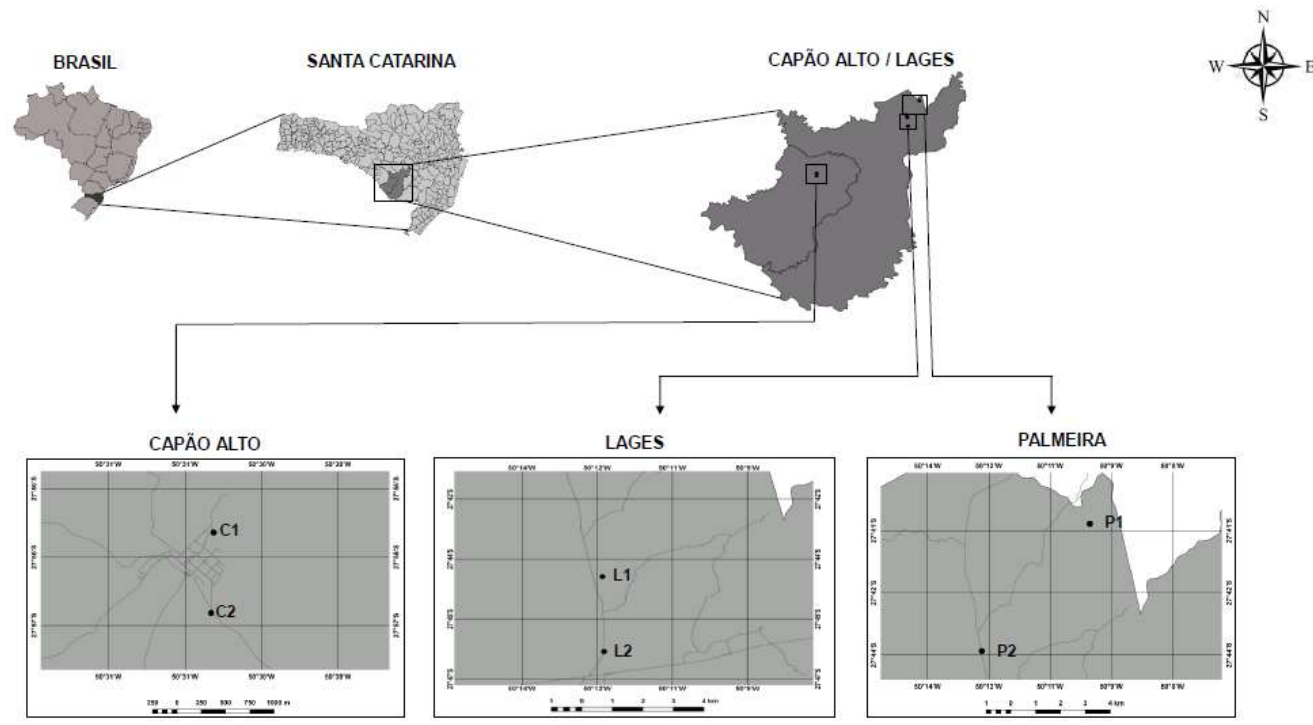

Figura 1. Locais de coleta nos municípios de Capão Alto (SC-390), Lages (BR-282) e Palmeira (SC 114). As coletas identificadas como C1, L1 e P1 ocorreram próximo a rodovias e os pontos C2, L2 e P2 são as testemunhas.

Figure 1. Collection sites in the municipalities of Capão Alto (SC-390), Lages (BR-282) and Palmeira (SC 114). The collections identified as $\mathrm{C} 1, \mathrm{~L} 1$ and $\mathrm{P} 1$ occurred near highways and points $\mathrm{C} 2, \mathrm{~L} 2$ and $\mathrm{P} 2$ are the witnesses. 


\subsection{Análise da Infusão}

As flores de macela foram separadas dos ramos e secas em estufa de circulação a $65^{\circ} \mathrm{C}$ até que não houvesse mais alteração de peso. O processo de infusão foi realizado segundo protocolo de Anderson (1999), onde utilizou-se $1 \mathrm{~g}$ de amostra das flores secas e moídas e $15 \mathrm{ml}$ de água ultrapura a $70^{\circ} \mathrm{C}$ por um tempo de 15 minutos. Após as amostras foram filtradas e receberam três gotas de ácido nítrico para a conservação (ANDERSON, 1999). A infusão foi realizada em duplicata.

A quantificação do teor de $\mathrm{Cu}, \mathrm{Mn}, \mathrm{Ni}$, e $\mathrm{Zn}$, na infusão foi realizada em Espectrometria de emissão óptica com plasma ICP-OES. Optima 8000® (Perkin Elmer), nas condições indicadas pelo fabricante. $\mathrm{O}$ teor de $\mathrm{Pb}$ na infusão foi determinado em espectrômetro de absorção atômica de alta resolução com fonte contínua (HR-CS AAS), com atomização em forno de grafite modelo ContrAA 700® (Analytik Jena), nas condições recomendadas pelo fabricante.

Foram realizadas oito provas em branco para o cálculo do Limite de Detecção Qualitativo (LDQ), segundo a equação $\mathrm{LDQ}=(\mathrm{M} \pm \mathrm{t} \times \mathrm{s})$, onde, $\mathrm{M}$ é a média das leituras para as provas em branco, t é o valor t-Student para um intervalo de confiança de $99 \%$, em razão do número de graus de liberdade de medições repetidas (3,1 no caso de oito repetições); e s é o desvio-padrão das leituras para provas em branco. Valores de LDQ encontram-se na Tabela 1.

Tabela 1. Valores de limite de detecção qualitativos (LDQ) de $\mathrm{Cu}$ $\mathrm{Mn}, \mathrm{Ni}, \mathrm{Zn}$ e $\mathrm{Pb}$ na infusão quantificada em ICP-OES e $\mathrm{Pb}$ quantificado em HR-CS-AAS.

Table 1. Qualitative detection limit values (LDQ) of $\mathrm{Cu}, \mathrm{Mn}, \mathrm{Ni}, \mathrm{Zn}$ and $\mathrm{Pb}$ in infusion quantified in ICP-OES and $\mathrm{Pb}$ quantified in $\mathrm{HR}$ CS-AAS.

\begin{tabular}{cllllll}
\hline & $\mathrm{Al}$ & $\mathrm{Cu}$ & $\mathrm{Mn}$ & $\mathrm{Ni}$ & $\mathrm{Pb}$ & $\mathrm{Zn}$ \\
\hline LDQ & 0,5 & 0,19 & 5,35 & 0,96 & 0,02 & 0,25
\end{tabular}

Fonte: próprio autor.

Os resultados foram submetidos à análise de variância conforme um modelo linear hierárquico com dois critérios de classificação (município e local de coleta). A homogeneidade de variâncias e a normalidade dos resíduos do modelo foram testadas por meio dos testes de Levenne e de Shapiro-Wilk. Para atenderem-se as pressuposições teóricas dos testes, houve necessidade de se aplicar a transformação logarítmica a algumas das variáveis analisadas. As análises foram realizadas com o uso do programa R (R CORE TEAM, 2016). Para todos os testes efetuados foi considerado o nível mínimo de significância de $5 \%$.

\section{RESULTADOS}

Em média a infusão das amostras de macela coletada as margens da BR 282 apresentaram os maiores teores de $\mathrm{Al}, \mathrm{Cu}$, $\mathrm{Ni}, \mathrm{Mn}$ e $\mathrm{Zn}$ (Tabela 2), o que pode estar relacionado ao maior volume tráfego de veículos e a proximidade com uma curva.

Os teores médios de Al variaram de 3,22 a 15,33 $\mathrm{mg} \mathrm{L}^{-1}$, sendo que, para as três rodovias os teores na infusão foram superiores as testemunhas (Tabela 2).

Os teores de Mn presentes na infusão de macela variaram de 172,43 a $533,78 \mathrm{mg} \mathrm{L}^{-1}$ (Tabela 2), sendo que, os maiores teores de $\mathrm{Mn}$ foram observados nas plantas coletadas as margens da SC - 390 (Capão Alto) e BR- 282 (Lages). Kalavrouziotis et al. (2007) avaliaram teores de Al, Pb, Cd, Zn, $\mathrm{Cu}, \mathrm{Ni}, \mathrm{Fe}, \mathrm{Mn}, \mathrm{Cr}, \mathrm{Co}, \mathrm{P}, \mathrm{Ca}, \mathrm{Mg}$ e $\mathrm{Na}$ de solo e plantas de milho as margens de rodovias na Grécia. Os autores observaram que as amostras de solo, parte aérea e raiz de milho apresentaram aumento dos teores de $\mathrm{Pb}, \mathrm{Mn}, \mathrm{Fe}$ e $\mathrm{Al}$ quando coletados a margem das rodovias.

Os teores de $\mathrm{Cu}$ e $\mathrm{Zn}$ na infusão de macela variaram de 2,0 a $5,1 \mathrm{mg} \mathrm{L}^{-1}$ e 14,87 a $44,25 \mathrm{mg} \mathrm{L}^{-1}$, respectivamente, sendo que os maiores teores foram observados na infusão das amostras coletadas as margens da BR - 282 (Tabela 2).

Os teores médios de Ni (Tabela 2) variaram entre 0,98 a $6,4 \mathrm{mg} \mathrm{L}^{-1}$, sendo que a infusão das plantas coletadas as margens da BR 282 apresentaram os maiores teores de Ni.

Os teores de $\mathrm{Pb}$ na infusão não apresentaram diferença significativa entre rodovias e nem entre locais de coleta. Os teores variaram entre $0,03 \mathrm{mg} \mathrm{L}^{-1}$ para plantas do Capão Alto e $0,05 \mathrm{mg} \mathrm{L}^{-1}$ para as de Lages e Palmeira.

Tabela 2. Teores médios de $\mathrm{Cu}, \mathrm{Mn}, \mathrm{Ni}, \mathrm{Pb}$ e $\mathrm{Zn}\left(\mathrm{mg} \mathrm{L}^{-1}\right)$ na infusão de plantas de Achyrocline satureioides coletadas em diferentes municípios, sem e com influência do tráfego rodoviário.

Table 2. Average levels of $\mathrm{Cu}, \mathrm{Mn}, \mathrm{Ni}, \mathrm{Pb}$ and $\mathrm{Zn}(\mathrm{mg} \mathrm{L}-1)$ in the infusion of Achyrocline satureioides plants collected in different municipalities, with and without influence of road traffic.

\begin{tabular}{lccccccc}
\hline Município & Local & $\mathrm{Al}$ & $\mathrm{Cu}$ & $\mathrm{Ni}$ & $\mathrm{Mn}$ & $\mathrm{Pb}$ & $\mathrm{Zn}$ \\
\hline Capão Alto & & & & & $\mathrm{mg} \mathrm{L}^{-1}$ & & \\
& testemunha & $7,2 \mathrm{~b}$ & $2,5 \mathrm{a}$ & $1,44 \mathrm{a}$ & $217 \mathrm{~b}$ & $0,033^{\mathrm{NS}}$ & $19,1 \mathrm{a}$ \\
Lages & $\mathrm{SC}-390$ & $15,2 \mathrm{a}$ & $2,0 \mathrm{a}$ & $0,98 \mathrm{a}$ & $372 \mathrm{a}$ & & $24,8 \mathrm{a}$ \\
& testemunha & $3,2 \mathrm{~b}$ & $3,3 \mathrm{~b}$ & $1,16 \mathrm{~b}$ & $357 \mathrm{~b}$ & $0,05^{\mathrm{NS}}$ & $14,8 \mathrm{~b}$ \\
\multirow{2}{*}{ Palmeira } & $\mathrm{BR}-282$ & $13,5 \mathrm{a}$ & $5,1 \mathrm{a}$ & $6,42 \mathrm{a}$ & $533 \mathrm{a}$ & & $44,2 \mathrm{a}$ \\
& testemunha & $4,2 \mathrm{~b}$ & $2,4 \mathrm{a}$ & $2,93 \mathrm{a}$ & $270 \mathrm{a}$ & $0,05^{\mathrm{NS}}$ & $27,4 \mathrm{a}$ \\
& $\mathrm{SC}-114$ & $15,3 \mathrm{a}$ & $3,2 \mathrm{~b}$ & $1,32 \mathrm{~b}$ & $172 \mathrm{a}$ & & $20,5 \mathrm{a}$ \\
\hline
\end{tabular}

Médias seguidas pela mesma letra na coluna, não apresentam diferenças ( $\mathrm{p}>0,05)$ entre locais dentre de cada município, pelo teste de Tukey.

\section{DISCUSSÃO}

Aumento do teor de Al na infusão pode estar relacionado a deposição de material particulado sobre a vegetação próximo as rodovias. Thorpe; Harrison (2008), apresentam um resumo das concentrações de metais presentes nas lonas de freio e emissão de poeira de freio no mundo e o teor de $\mathrm{Al}$ emitido na forma de poeira de freio alcança $2500 \mathrm{mg} \mathrm{kg}^{-1}$. A deposição seca dessas poeiras ocorre as margens das rodovias e seu alcance depende da direção e velocidade dos ventos.
Segundo Kibblewhite (2018) os contaminantes presentes nas emissões dos veículos e no desgaste de veículos e rodovias são transferidos para o solo e plantas por partículas transportadas pelo ar, poeira, respingos, pulverização e escoamento da precipitação pluviométrica. Esse autor afirma que a contaminação é maior perto da borda da rodovia, mas pode se estender a distâncias superiores a $50 \mathrm{~m}$ e que os principais elementos inorgânicos incluindo $\mathrm{As}, \mathrm{Cd}, \mathrm{Cr}, \mathrm{Cu}, \mathrm{Pb}$, Ni, Zn. 
Hong et al. (2018) avaliaram os teores de metais em água de escoamento superficial em rodovia em Shenzhen, China. Esses autores afirmaram que o sistema de exaustão dos veículos foi a principal fonte dos metais $\mathrm{Cr}, \mathrm{Cu}, \mathrm{Pb}, \mathrm{Ni}$, e $\mathrm{Zn}$ e que seria muito importante considerá-los em termos de controle da poluição por metais pesados em superfícies de estradas urbanas. Hong et al. (2018) indicaram ainda que a queima da gasolina foi a principal fonte de $\mathrm{Cr}, \mathrm{Ni}$ e $\mathrm{Pb}$, enquanto o desgaste de frenagem e o desgaste dos pneus foram as principais fontes de $\mathrm{Cu}$ e $\mathrm{Zn}$, respectivamente.

Jin et al. (2007) avaliaram os fatores não edáficos que contribuíram para o teor de $\mathrm{Pb}$ em chá comercial e observaram que o teor de $\mathrm{Pb}$ nas folhas de chá verde estava positivamente correlacionada com o nível de industrialização de um distrito ( $\mathrm{R}=0,83$, o nível significativo em $\mathrm{P}<0,05$. Além disso, quantidades maiores de $\mathrm{Pb}$ foram observadas em folhas de plantas que crescem perto de rodovias do que aquelas que crescem mais longe. Indicando que a atividade automobilística foi outro provável contribuinte para o acúmulo de chumbo no chá.

Para avaliar o potencial de risco a saúde optou-se por construindo um cenário hipotético em que um adulto ingeriria de $250 \mathrm{ml}$ da infusão de macela coletada as margens da BR 282 no município de Lages e compará-los aos valores apresentados na Portaria no 2.914 do Ministério da Saúde, que dispõe sobre os procedimentos de controle e de vigilância da qualidade da água para consumo humano e seu padrão de potabilidade (BRASIL, 2011).

Para Al, a infusão apresentou teor superior ao valor máximo permitido (VMP) (Tabela 3). O Alumínio tem efeito neurotóxico, e é considerado muito tóxico a pacientes em diálise, devido à insuficiência renal crônica, embora também existam relatos de neuro e osteotoxicidade semelhantes também em pacientes sem insuficiência renal (FLATEN, 2002). A exposição ao Al representa um problema de saúde para a população em geral. Entretanto, a questão mais controversa é se a exposição ao $\mathrm{Al}$ está associada à doença de Alzheimer, embora existam evidências consideráveis para tal relação, ainda está em aberto o debate sobre o nexo causal (FLATEN, 2002).

Tabela 3. Al, $\mathrm{Cu}, \mathrm{Mn}, \mathrm{Ni}, \mathrm{Pb}$ e $\mathrm{Zn}$ em $250 \mathrm{ml}$ de infusão de macela e valores máximos permitidos (VMP) pela Portaria $n^{\circ} 2.914$ do Ministério da Saúde (BRASIL, 2011).

Table 3. $\mathrm{Al}, \mathrm{Cu}, \mathrm{Mn}, \mathrm{Ni}, \mathrm{Pb}$ and $\mathrm{Zn}$ in $250 \mathrm{ml}$ of macela infusion and maximum allowed values (MPV) by Ministry of Health Ordinance No. 2.914 (BRAZIL, 2011).

\begin{tabular}{lcc}
\hline Elemento & Infusão & $\mathrm{VMP}^{* *}$ \\
\hline & $250 \mathrm{ml}$ & $\mathrm{mg} \mathrm{L}^{-1}$ \\
$\mathrm{nyl}$ & 3,36 & 0,2 \\
$\mathrm{Cu}$ & 1,3 & 0,2 \\
$\mathrm{Mn}$ & 133,44 & 0,1 \\
$\mathrm{Ni}$ & 1,6 & 0,07 \\
$\mathrm{~Pb}$ & 0,01 & 0,01 \\
$\mathrm{Zn}$ & 11,06 & 5,0 \\
\hline
\end{tabular}

O teor de Mn foi muito superior ao VMP (Tabela 3). Segundo Nordberg et al. (2007) as concentrações de Mn tendem a ser mais elevada em alimentos de origem vegetal, como por exemplo os chás. Alguns trabalhos descritos na literatura indicam uma elevada concentração de $\mathrm{Mn}$ em infusões de plantas medicinais (AMARANTE et al., 2011). Segundo Fiorini (2008) o Mn é constituinte de diversas enzimas e atua como ativador de várias outras. Participa do metabolismo dos carboidratos, aminoácidos e colesterol, é antioxidante e faz parte da formação da cartilagem e ossos. Em doses elevadas, o Mn é neurotóxico, alguns sintomas característicos desse quadro são alterações de humor, agitação, depressão, dificuldade de processar informações, e principalmente sintomas de parkinsonianos (tremores e rigidez corporal) (ASCHNER et al., 2007).

$\mathrm{O}$ teor de Ni em $250 \mathrm{ml}$ de infusão (Tabela 3) apresentouse superior ao VMP. Entretanto o Ni é considerado um constituinte normal das dietas e seus compostos são geralmente reconhecidos como seguros, quando, usado como ingrediente direto na alimentação humana (CEMPEL; NIKEL, 2006). Em humanos $27 \%$ de $\mathrm{Ni}$ é absorvido quando da ingestão de água contaminada, enquanto que em alimentos apenas $0,7 \%$ da dose de Ni é absorvida (diferença de 40 vezes) (SUNDERMAN et al., 1989, CEMPEL; NIKEL, 2006). Em geral, devido à lenta absorção de compostos de Ni pelo trato gastrointestinal, esses não são considerados tóxicos.

$\mathrm{O}$ teor de $\mathrm{Pb}$ em $250 \mathrm{ml}$ de chá é equivalente ao VMP para água potável (BRASIL, 2011). Para Karak e Bhagat (2010) infusões que contenham teores de $\mathrm{Pb}$ que excedem o limite estabelecido pela Organização Mundial da Saúde para água potável de 0,05 mg L-1 (WHO, 2003) oferecem risco a saúde a longo prazo. A presença de $\mathrm{Pb}$ no organismo humano interfere na produção da hemoglobina, causa danos renais (nefrite) e neurológicos (plumbismo) (BROWN et al., 2012; USEPA, 2017). O Pb é transferido através da membrana placentária e acumula-se nos tecidos fetais, sendo que exposição pré-natal ao $\mathrm{Pb}$ tem sido associada ao aumento do número de abortos, anomalias fetais e atraso no crescimento uterino. $\mathrm{O} \mathrm{Pb}$ tem sido relacionado a hiperatividade infantil, déficit de atenção, menor quociente de inteligência (QI) e criminalidade na adolescência. A estimativa de queda de inteligência é de 1 a 3 pontos de QI para cada aumento de 0,1 $\mathrm{mg}$ L-1 de Pb no sangue em crianças (USEPA, 2017). Estudo realizado no Brasil demonstrou haver forte associação entre a exposição ao chumbo e o comportamento antissocial em adolescentes (OLYMPIO et al., 2010).

Os teores obtidos para os elementos avaliados neste estudo podem indicar que o consumo de chá de macela coletada a margens, de rodovias como a BR-282, pode resultar em risco a saúde humana, principalmente, nos casos em que há substituição da água pela infusão do chá.

\section{CONCLUSÕES}

Em média a infusão das amostras de macela coletada as margens da BR 282 apresentaram os maiores teores de $\mathrm{Al}, \mathrm{Cu}$, $\mathrm{Ni}, \mathrm{Mn}$ e Zn;

Não houve diferença estatística entre teores de $\mathrm{Pb}$ em macelas coletadas nas diferentes rodovias e as testemunhas;

O consumo de chá de macela coletada próximo a rodovias pode resultar em risco a saúde humana.

\section{REFERÊNCIAS}

AKBAR, K. F.; HEADLEY, A. D.; HALE, W. H. G.; ATHAR, M. Heavy metal contamination of roadside soils of Northern England. Soil and Water Research, v. 1, n. 4, p. 158-163, 2006.

AKISUE, M. K. Análise do óleo essencial de Achyrocline satureioides DC. Compositae. Revista Farmácia Bioquímica Universidade de São Paulo, São Paulo, v. 9 , p. 107-114, 1971. 
AMARANTE, C. B.; Silva, F. C.; MUlleR, C. S.; MULLER, A. H. Avaliação da composição mineral do chá da folha senescente de Montrichardia linifera (arruda) schott (Araceae) por espectrometria de absorção atômica com chama (faas). Revistas Quim. Nova, v. 34, n. 3, p. 419-423, 2011.

ANDERSON, K. A. Analytical techniques for inorganic contaminants. Gaithersburg: AOAC International, 1999. $316 \mathrm{p}$.

ARSLAN, H.; GIZIR, A. M. Heavy-metal content of roadside soil in Mersin, Turkey. Fresenius Environ Bulletin, v. 15, p. 15-20, 2006.

ASCHNER, M.; GUILARTE, T. R.; SCHNEIDER, J. S.; ZHENG, W. Manganese: recent advances in understanding its transport and neurotoxicity. Toxicology and Applied Pharmacology, San Diego, v. 221, n. 2, p. 131-147, 2007. https://dx.doi.org/10.1016/j.taap.2007.03.001

DOI:

BAKER, J. M.; OCHSNER, T. E.; VENTEREA, R. T.; GRIFFIS, T. J. Tillage and soil carbon sequestration What do we really know? Agriculture, Ecosystems and Environment, v. 118, p. 1-5, 2007. DOI: 10.17221/66/2016-PSE

BARBOSA, A. E.; HVITVED-JACOBSEN, T. Highway runoff and potential for removal of heavy metals in an infiltration pond in Portugal. Science Total Environment, v. $235, \quad$ n. $1-3, \quad$ p. $151-159,1999$. DOI: https://dx.doi.org/10.1016/S0048-9697(99)00208-9

BOHLMANN, F.; BURKHARDT, T.; ZDERO, C. Naturally Occurring Acetylenes. Academic Press: London, 1973. $547 \mathrm{p}$

BRANDELLI, C. L. C.; GIORDANI, R. B.; DE CARLI, G. A.; TASCA, T. Indigenous traditional medicine: in vitro anti-giardial activity of plants used in the treatment of diarrea. Parasitology Research, Berlin v. 104, n. 6, p. 1345-1349, 2009.

DOI: https://dx.doi.org/10.1007/s00436-009-1330-3

BRASIL MINISTÉRIO DA SAÚDE. Portaria n. ${ }^{\circ}$ 2.914, de 12 de Dezembro de 2011. Dispõe sobre normas de potabilidade de água para o consumo humano. Brasília: SVS, 2011

BROUSSALIS A. M.; FERRARO, G. E.; GURNI, A.; COUSSIO, J. D. Phenolic constituents of four Achyrocline species. Biochemical Systematics and Ecology, Oxford, v. 16, p. 401-402, 1988.

BROWN, M. J.; MARGOLIS, S. Lead in Drinking Water and Human Blood Lead Levels in the United States. Centers for Disease Control and Prevention, v. 61, n. 4, p. 1-9, 2012.

CEMPEL, M.; NIKEL, G. Nickel: a review of its sources and environmental toxicology. Polish Journal of Environmental Studies, v. 15, n. 3, p. 375-382, 2006.

DIÁRIO CATARINENSE. Catarinenses seguem a tradição de colher a macela na Sexta-feira Santa. 2013. Disponível em: $<$ http://dc.clicrbs.com.br/sc/noticias/noticia/2013/03/catar inenses-seguem-a-tradicao-de-colher-a-macela-na-sextafeira-santa-4090143.html>. Acesso em: 27 outubro 2018.

DOLAN, M. S.; CLAPP, C. E.; ALLMARAS, R. R.; BAKER, J. M.; MOLINA, J. A. E. Soil organic nitrogen in a Minnesota soil as related to tillage, residue, and nitrogen management. Soil Tillage Research, v. 89, p. 221-231, 2006.
FERRARO, G. E.; NORBEDO, C.; COUSSIO, J. D. Polyphenols from Achyrocline satureioides. Phytochemistry, Oxford, v. 20, n. 8, p. 2053-2054, 1981. DOI: https://dx.doi.org/10.1016/0031-9422(81)84074-5

FIORINI, L. S. Dossiê: Os minerais na alimentação. Rev-fi, Food Ingredients Brasil. n. 4, 2008. Disponivél em: < http://www.revista-fi.com/materias/52.pdf $>$ Acesso em 22 de outubro de 2019.

FLATEN, T. Aluminium in tea - concentrations, speciation and bioavailability. Coordination chemistry reviews, Amsterdam, v. 228, n. 2, p. 385-395, 2002. DOI: https://dx.doi.org/10.1016/S0010-8545(02)00036-X

HJORTENKRANS, D. S. T.; BERGBÄCK, B. G.; HÄGGERUD, A. V. Metal emissions from brake linings and tires: Case studies of Stockholm, Sweden 1995/1998 and 2005. Environmental Science Technology, Easton, v. 41, n. 15, p. 5224-5230, 2007. DOI: https://dx.doi.org/10.1021/es070198o

HONG, N.; ZHU, P.; LIU, A.; ZHAO, X.; GUAN, Y. Using an innovative flag element ratio approach to tracking potential sources of heavy metals on urban road surfaces. Environmental Pollution, Barking v. 243, p. 410-417, 2018.

DOI: https://dx.doi.org/10.1016/j.envpol.2018.08.098

JIN, I. W.; HE, F. Y.; ZHANG, K.; ZHOU, G. D.; SHIL, L. J.; ZHENG, S. J. Lead contamination in tea leaves and nonedaphic factors affecting it. Chemosphere, Oxford, v. 61, n. $5, \quad$ p. 726-732, 2005. DOI: https://dx.doi.org/10.1016/j.chemosphere.2005.03.053

KALAVROUZIOTIS, I. K.; CARTER, J.; VARNAVAS, S. P.; MEHRA, A.; DRAKATOS, P. A. Towards an understanding of the effect of road pollution on adjacent food crops: zea mays as an example. International Journal of Environment and Pollution, Geneva, v. 30, n. 3-4, 2007.

DOI: https://dx.doi.org/10.1504/IJEP.2007.014830

KARAK, T.; BHAGAT, R. M. Trace elements in tea leaves, made tea and tea infusion: a review. Food Research International, Barking, v. 43, n. 9, p. 2234-2252, 2010. DOI: https://dx.doi.org/10.1016/j.foodres.2010.08.010

$\mathrm{KOCH}, \mathrm{V}$. Estudo Etnobotânico das Plantas Medicinais na Cultura Ítalobrasileira no Rio Grande do Sul - Um Modelo Para o Cultivo Comercial na Agricultura Familiar. 2000. 152f. Dissertação (Mestrado) Universidade Federal do Rio Grande do Sul, Porto Alegre, 2000.

LAMATY, G.; BÈSSIERE, J. M.; SCHENKEL, E. P.; SANTOS, M.; BASSANI, V. L. The chemical composition of some Achyrocline satureioides and Achyrocline alata oils from Brazil. Brazilian Journal of Essential Oil Research, v. 3, n. 5, p. 317-332, 1991. DOI: https://dx.doi.org/10.1080/10412905.1991.9697951

LEE, J. Y.; KIM, H.; KIM, Y.; HAN, M. Y. Characteristics of the event mean concentration (EMC) from rainfall runoff on an urban highway. Environmental Pollution, Barking, v. 59, n. 4, p. 884-888, 2011. DOI: https://dx.doi.org/10.1016/j.envpol.2010.12.022

LOUGH, G. C.; CHAUER, J. J.; PARK, J. S.; SHAFER, S. M.; DEMINTER, J; WEINSTEIN, J. Emissions of metals associated with motor vehicle roadway. Environmental Science Technology, Easton, v. 39, n. 3, p. 826-836, 2005. DOI: https://dx.doi.org/10.1021/es048715f 
MCKENZIE, E. R.; MONEY, J. E.; GREEN, P. G.; YOUNG, T. M. Metals associated with stormwater - relevant brake and tire samples. Science of The Total Environment, Amsterdam, v. 407, n. 22, p. 5855-5860, 2009. DOI: https://dx.doi.org/10.1016/j.scitotenv.2009.07.018

MONKS, P. S.; GRANIER, C.; FUZZI, S.; STOHL, A.; WILLIAMS, M.L.; AKIMOTO, H. Atmospheric composition change-global and regional air quality. Atmospheric Environment, Oxford, v. 43, n. 33, p. 52685350, 2009.

DOI: https://dx.doi.org/10.1016/j.atmosenv.2009.08.021

NORDBERG G. F.; FOWLER, B.; NORDBERG, M. Handbook on the Toxicology of Metals. Third Edition. 2007. ISBN: 9780123973399

OLYMPIO, K. P. K.; OLIVEIRA, P. V.; NAOZUKA, J.; CARDOSO, M. R. A.; MARQUES, A. F.; GUNTHER, W. M. R.; BECHARA, E. J. H. Surface dental enamel lead levels and antisocial behavior in Brazilian adolescents. Neurotoxicology and Teratology, Oxford, v. 32, n. 2, p. 273-279, 2010

DOI: https://dx.doi.org/10.1016/j.ntt.2009.12.003

PAGOTTO, C.; REMY, N.; LEGRET. M.; LE CLOIREC, P.; Heavy metal pollution of road dust and roadside soil near a major rural highway. Environmental Technology, London, v. 22, n. 3, p. 307-319, 2001. DOI: https://dx.doi.org/10.1080/09593332208618280

PETROVICK, P. R.; ORTEGA, G.; BASSANI, V. L. From a medicinal plant to a pharmaceutical dosage form. A (still) long way for the Brazilian medicinal plants. Journal of the Brazilian Association Advancement Science, v. 49, p. 364-369, 1997.

PIRKER, H.; HASELMAIR, R.; KUHN, E.; SCHUNKO, C.; VOGL, C. R. Transformation of traditional knowledge of medicinal plants: the case of Tyroleans 135 (Austria) who migrated to Australia, Brazil and Peru. Journal of Ethnobiology and Ethnomedicine, v. 8, n. 44, 2012. DOI: https://dx.doi.org/10.1186/1746-4269-8-44

R CORE TEAM. R: A language and environment for statistical computing. $\mathrm{R}$ Foundation for Statistical Computing, Vienna, 2016. Disponível em://www.Rproject.org/.

RITTER, M. R.; SOBIERAJSKI, G. R.; SCHENKEL, E. P.; MENTH, L. A. Plantas usadas como medicinais no município de Ipê, RS, Brasil. Revista Brasileira de Farmacognosia, Maringá, v. 12, n. 2, p. 51-62, 2002. DOI: http://dx.doi.org/10.1590/S0102-695X2002000200001

SANTA CATARINA 24 HORAS. BR 282: Um sonho de 200 anos. Disponível em: $<$ http://www.santacatarina24horas.com/colunas/opini ao/12758-br-282-um-sonho-de-200-anos.html>. Acesso em: 10 de outubro de 2018.

SIMÕES, C. M.; SCHENKEL, E. P.; BAUER, L.; LANGELOH, A. Pharmacological investigations on Achyrocline satureioides (Lam.) DC, Compositae. Journal of Ethnopharmacology, v. 22, n. 3, p. 281-293, 1988.

THORPE, A.; HARRISON, R. Sources and properties of nonexhaust particulate matter from road traffic: a review. Science of The Total Environment, Amsterdam, v. 400, n. $1-3, \quad$ p. 270-282, 2008. DOI: https://dx.doi.org/10.1016/j.scitotenv.2008.06.007

USEPA. US. Environmental Protection Agency, 2017. EPA Integrated Risk Information System. Disponível em: $<$ https://www.epa.gov/lead/learn-about-lead\#exposure $>$ Acesso em: 22 de outubro de 2019.

WARD, N. I. Multielement contamination of British motorway environments. Science of The Total Environment, Amsterdam, v. 93, p. 393-401, 1990. DOI: https://dx.doi.org/10.1016/0048-9697(90)90130-M

WERKENTHIN, M.; KLUGE, B.; WESSOLEK, G. Metals in European roadside soils and soil solution - A review. Environmental Pollution, v. 189, p. 98-110, 2014. DOI: https://dx.doi.org/10.1016/j.envpol.2014.02.025

WHO_WORLD HEALTH ORGANIZATION. Lead in drinking-water, background document for preparation of WHO Guidelines for drinking-water quality. 2003.

IKENAKA, Y.; NAKAYAMA, S.; MUZANDU, K.; CHOONGO, K.; TERAOKA, H.; MIZUNO, N.; ISHIZUKA, M. Heavy metal contamination of soil and sediment in Zambia. African Journal of Environmental Science and Technology, v. 4, n. 11, p. 729-739. 2010. DOI: $10.5897 / A J E S T 10.179$ 